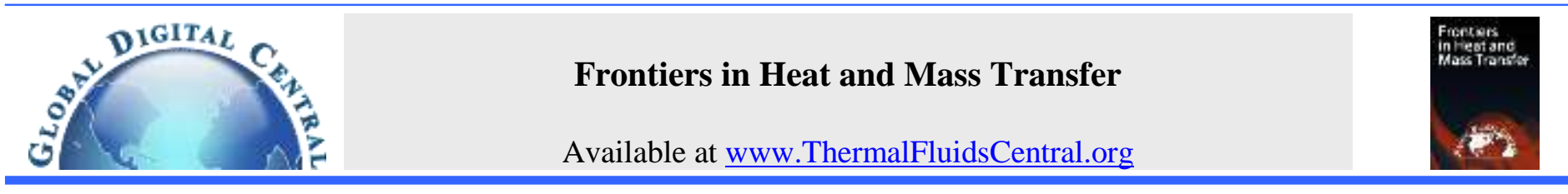

\title{
NUMERICAL SIMULATION OF NATURAL CONVECTION IN RECTANGULAR CAVITIES WITH DIFFERENT ASPECT RATIOS
}

\author{
Olanrewaju M. Oyewola ${ }^{\mathrm{a}, \mathrm{b}^{*}}$, Samuel I. Afolabi ${ }^{\mathrm{b}}$, Olawale S. Ismail ${ }^{\mathrm{b}}$ \\ a School of Mechanical Engineering, Fiji National University, Suva, Fiji \\ ${ }^{b}$ Department of Mechanical Engineering, University of Ibadan, Ibadan, 200213, Nigeria
}

\begin{abstract}
The problem of natural convection in rectangular cavities with different aspect ratios has been numerically analyzed in this study. Cavities considered have their right vertical walls heated and cooled at the opposite with constant temperatures, while horizontal walls are kept adiabatic. The objective of this study is to ascertain the significant effects of Rayleigh numbers (Ra), Nusselt numbers (Nu) and aspect ratios (AR) on flow and heat transfer in rectangular cavities. The equations of Navier-Stokes and energy are solved by applying Galerkin weighted residual Finite Element Method. Parametric calculations are performed for Rayleigh numbers (Ra) ranging from $10^{4}$ to $10^{8}$ and for aspect ratios (AR=0.25, 0.5, 1 and 2). Variations of Streamlines and Isotherms contours of the resulting flow fields and temperatures are presented in correlation with the estimated Nusselt numbers (Nu) for a fluid measured at air room temperature having Prandtl number (Pr) of 0.71. The results of the numerical simulation reveals that at higher Rayleigh number of flow, heat transfer in rectangular cavities increases. The Nusselt number estimated close to the boundary of the hot wall indicate a predominant heat transfer by convection in higher AR, hence, results indicates that with increasing aspect ratio, the thermal boundary layer thickness were steadily increased thereby contributing to major heat transfer of fluid by convection in the rectangular cavities. The engineering application of natural convection in cavities such as thermal storage facilities has made this study significant.
\end{abstract}

Keywords: Cavity, fluid flow, Galerkin finite element, heat transfer, natural convection.

\section{INTRODUCTION}

The subject of natural convection flows in rectangular cavities has been investigated for several decades due to its vital significance in many natural and industrial operations. Results emanated from such studies have greatly contributed to the advancement and effective utilization of computational processes and a range of applications in engineering which include thermal insulation, nuclear reactors, mechanical and electronic equipment cooling, energy storage facilities and ventilation Raisi and Arvin, (2018). Due to the importance of convection heat transfer in engineering system, several researches have been conducted on natural convection within cavities with various boundary conditions in order to achieve certain target, Mahmud and Rawand, (2020).

Souda et al, (2017) numerically studied free convection in rectangular cavities with different aspect ratios in order to determine fluid friction and entropy generation distribution due to heat transfer mechanism for Rayleigh numbers from $10^{5}$ to $10^{8}$ and aspect ratio varied from $3,5,7$ and 12 by applying the momentum equations. It was found that the rate of increase in Rayleigh numbers to the total entropy generation strongly influences the aspect ratios. From the Experimental view, it has been revealed that when cavity is heated at the bottom wall and cooled at the top wall, the increase in Rayleigh number led to high average heat transfer coefficient. Therefore, Nusselt number in vertical cavity was observed to strongly depend on aspect ratios, Prandtl number, Rayleigh number and boundary conditions of the sidewalls, Ali et al, (2018).

Moreover, Patil and Singh, (2017) numerically studied lamina free convection within a uniformly heated close cavity. It was found that Rayleigh number was increased at fixed aspect ratio thereby led to an increase in the Nusselt number at the region of the hot wall. With the efforts of Saddam, (2020), a study on significant effect of variable viscosity along a vertical cylinder enclosed in non-Darcy porous medium raised a conclusion that heat transfer rate increases remarkably at high values of viscosity for gases and liquids, in the same manner as gases increases for every decrease in mass transfer rate. Elyazid et al, (2021) focus their study on natural convection heat transfer along isothermal vertical plate enclosed in saturated porous non-Darcian medium, they found buoyancy forces to have increased the boundary layer cooling and thickness of the thermal boundary layer was drastically minimized.

Further, the results of convection heat transfer for various aspect ratios and Rayleigh number were obtained to develop a useful correlation for average Nusselt number inside the cavity. Hassan et al, (2019) obtained a significant change in a fluid behavior at a maximum inclination angle when they performed a numerical study in a parallelogram cavity using $\mathrm{Cu}$-water as nanofluid, the heat transfer was enhanced and conclusion was reached that nanofluid concentration significantly affected the heat transfer rate. Numerical solution of Santhi et al, (2021) on the effects of heat and mass transfer in non-steady MHD natural convection over an infinite vertical plate shows that velocity flow enhances the magnetic parameter and permeable nature of the porous medium. Also, the increment of the magnetic parameter led to an increase in the skinfriction considered.

Recently, numerical studies on conjugate free convection in porous media, inclined thin baffles in cavities, nanofluid filled and magnetic field within rectangular cavities have demonstrated that Rayleigh numbers are predominant factor in fluid flows and heat transfer characteristics Mohamed et al, (2020); Abdulkadhim et al,(2018);

*Corresponding author. Email. olanrewaju.oyewola@fnu.ac.fj 
Selimefendigil and Öztop, (2016); Aydin and Yang, (2000). Barik and Khaled, (2019). It worth noting that Lo et al, (2004) considered natural convection in cavities with insulated horizontal walls while the vertical walls are heated and cooled at the opposite. The emanated results showed that by varying the cavity aspect ratios, Rayleigh number and vorticity were drastically increased. Similarly, Mazgar et al, (2019) concluded that radiation has a strong impact on the rising of vortices and homogenous impact on field of temperature in their work on the effect of gas radiation on laminar free convection within a square cavity having internal heat generation.

Despite numerous studies conducted on natural convection in cavities by experimental and numerical approximations, thermally driven flows in rectangular cavities with different aspect ratios in a differentially heated vertical walls and adiabatic horizontal walls has not been given considerable attentions. This might partly due to the complexity of the problem as well as other associated issues. It worth noting that contribution in this area might improve our understanding and lead to better system design and performance especially in the useful applications such as drying and storage processes phenomenon. In addition, the program validated codes can be used to test for the equations of Navier-Stokes in steady, laminar and incompressible flows. Hence, this work focuses on numerical data analysis of natural convection heat transfer within rectangular cavities having hot right walls and cold left walls of Rayleigh numbers ranging from $10^{4}$ to $10^{8}$ and aspect ratios of $0.25,0.5,1$ and 2 .

\section{PROBLEM STATEMENT}

The cavity illustrated in Fig. 1 is chosen for simulating natural convective flow and heat transfer characteristics while the gravitational force is acting downwards, the aspect ratio of the cavity is defined as the ratio of width to height. Right wall is heated and the left wall is cooled while the top and bottom walls are kept adiabatic. Temperatures are assumed to be constant throughout the process. Heat transfer between two vertical walls of different surface temperatures in a cavity can be performed with the mode of conduction, convection and radiation. However, conduction and convection are the two modes considered in this study while radiation is assumed negligible. By varying the Rayleigh numbers between $10^{4}$ to $10^{8}$ for aspect ratios of $0.25,0.5,1$ and 2 , the velocity and temperature parameters can be analyzed.

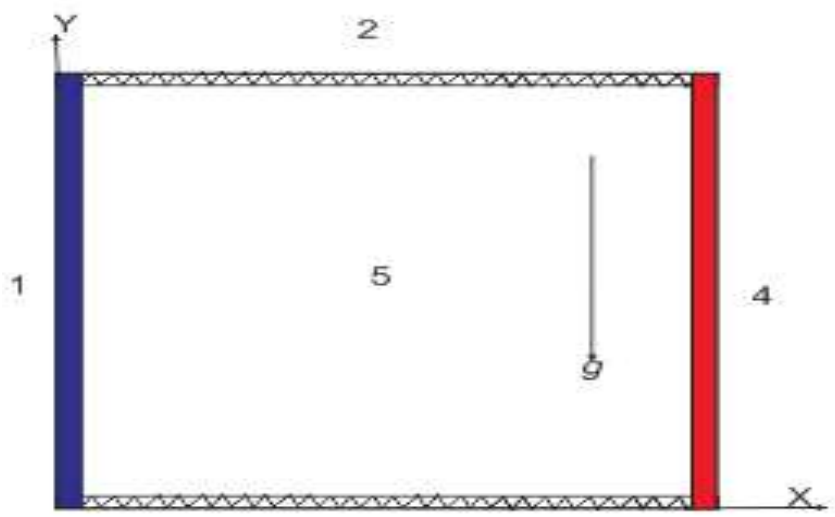

3

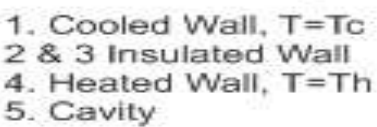

Fig. 1 The Cavity physical model

\section{MATHEMATICAL FORMULATION}

The governing equations for this problem are the differential equations of Navier-Stokes and energy applied to a two-dimensional, steady state, laminar, incompressible flows and Newtonian equations. The Boussinesq approximation which relates density gradient to temperature gradient is employed in formulating buoyancy force in the momentum equation. Neglecting the viscous dissipation term in the energy equation, the resulting equations that governs the present flows can be expressed as follows:

$$
\begin{aligned}
& \frac{\partial u}{\partial x}+\frac{\partial v}{\partial y}=0 \\
& u \frac{\partial u}{\partial x}+v \frac{\partial u}{\partial y}=-\frac{1}{\rho} \frac{\partial P}{\partial x}+\vartheta\left\{\frac{\partial^{2} u}{\partial x^{2}}+\frac{\partial^{2} u}{\partial y^{2}}\right\} \\
& u \frac{\partial v}{\partial x}+v \frac{\partial v}{\partial y}=-\frac{1}{\rho} \frac{\partial P}{\partial y}+\vartheta\left\{\frac{\partial^{2} v}{\partial x^{2}}+\frac{\partial^{2} v}{\partial y^{2}}\right\}+\beta g\left(T_{h}-T_{c}\right) \\
& u \frac{\partial T}{\partial x}+v \frac{\partial T}{\partial y}=\propto\left(\frac{\partial^{2} T}{\partial x^{2}}+\frac{\partial^{2} T}{\partial y^{2}}\right)
\end{aligned}
$$

Introducing the stream function $\psi(x, y)$, continuity equation is satisfied and then eliminated.

$$
u=\frac{\partial \psi}{\partial y}, \quad v=-\frac{\partial \psi}{\partial x}
$$

The vorticity equation $\omega$ is derived by involving the stream function in terms of $u$ and $v$.

$$
\omega=\frac{\partial v}{\partial x}-\frac{\partial u}{\partial y}
$$

Dimensionless variables below are applied to represent and calculate its stability criteria. Saddam, (2020)

$$
\begin{aligned}
& \Psi=\frac{\psi}{\alpha}, \quad \mathrm{X}=\frac{x}{\delta}, \quad \Omega=\frac{\omega \delta^{2}}{\alpha}, \\
& \mathrm{Y}=\frac{y}{\delta}, \quad \mathrm{U}=\frac{\mathrm{u} \delta}{\alpha}, \quad \mathrm{V}=\frac{\mathrm{v} \delta}{\alpha}, \\
& \theta=\frac{T-T_{c}}{T_{h}-T_{c}}, \quad \operatorname{Pr}=\frac{\vartheta}{\alpha} \\
& \mathrm{Ra}=\frac{\beta g\left(T_{h}-T_{c}\right) \delta^{3} P r}{\vartheta^{2}}, \quad N u=\frac{h l}{k}
\end{aligned}
$$

The vorticity transport equation for dimensionless variables is defined as

$U \frac{\partial \Omega}{\partial X}+V \frac{\partial \Omega}{\partial Y}=\operatorname{Pr}\left(\frac{\partial^{2} \Omega}{\partial X^{2}}+\frac{\partial^{2} \Omega}{\partial Y^{2}}\right)+\operatorname{Ra} \cdot \operatorname{Pr} \frac{\partial \theta}{\partial X}$

The dimensionless form of vorticity equation in terms of stream function is transformed to;

$\Omega=-\left(\frac{\partial^{2} \Psi}{\partial X^{2}}+\frac{\partial^{2} \Psi}{\partial Y^{2}}\right)$

The dimensionless form of energy equation becomes

$U \frac{\partial \theta}{\partial X}+V \frac{\partial \theta}{\partial Y}=\frac{\partial^{2} \theta}{\partial X^{2}}+\frac{\partial^{2} \theta}{\partial Y^{2}}$ 
Since there is no-slip boundary conditions on the walls of the cavities, the no-slip condition results in zero velocity at the wall.

\section{Velocity boundary;}

$\boldsymbol{u}(\boldsymbol{x}, 0)=\boldsymbol{u}(\boldsymbol{x}, y)=\boldsymbol{u}(\mathbf{0}, y)=\boldsymbol{u}(x, y)=\mathbf{0}$

$\boldsymbol{v}(x, 0)=\boldsymbol{v}(x, y)=\boldsymbol{v}(0, y)=\boldsymbol{v}(x, y)=\mathbf{0}$

Temperature boundary;

For left and right walls respectively,

$T(0, y)=T_{c}, T(x, y)=T_{h}$

For top and bottom wall respectively

$\boldsymbol{T}(x, \boldsymbol{y})=\boldsymbol{T}(x, \mathbf{0})=\mathbf{0}$

\subsection{Method of Solution}

The dimensionless equations subject to boundary conditions are solved using the finite element method based on Galerkin weighted residual formulation. For a typical finite element inside the cavity, the unknowns are $u, v, \Omega$ and $\theta$. The basis set of interpolation function used is defined as $\left\{\varphi_{k}\right\}_{k=1}^{N}$ for

$u=\sum_{k=1}^{N} \varphi_{k}(x, y) \cdot u_{k}$,

$v=\sum_{k=1}^{N} \varphi_{k}(x, y) \cdot v_{k}$

$\Omega=\sum_{k=1}^{N} \varphi_{k}(x, y) . \Omega_{k}$,

$\theta=\sum_{k=1}^{N} \varphi_{k}(x, y) \cdot \theta_{k}$

The nonlinear residual dimensionless equations in (8) and (10) are defined as

$$
\begin{aligned}
& R_{i}^{(1)}=\left[\sum_{k=1}^{N} \Omega_{k} \int_{A^{\prime \prime}}\left[\sum_{k=1}^{N}\left(\varphi_{k} \cdot u_{k}\right) \frac{\partial \varphi_{k}}{\partial X}+\left(\sum_{k=1}^{N}\left(\varphi_{k} \cdot v_{k}\right) \frac{\partial \varphi_{k}}{\partial Y}\right)\right] \varphi_{i} d x d y\right]-\operatorname{Pr} \sum_{k=1}^{N} \Omega_{k} \int\left[\frac{\partial \varphi_{i}}{\partial A^{*}} \frac{\partial \varphi_{k}}{\partial X}+\frac{\partial \varphi_{i}}{\partial Y} \frac{\partial \varphi_{k}}{\partial Y}\right] d x d y \\
& -\operatorname{RaPr} \sum_{k=1}^{N} \theta_{k} \int_{A^{*}} \frac{\partial \varphi_{k}}{\partial X}=0
\end{aligned}
$$

$R_{i}^{(2)}=\left[\sum_{k=1}^{N} \theta_{k} \int_{A^{(e)}}\left[\sum_{k=1}^{N}\left(\varphi_{k} \cdot u_{k}\right) \frac{\partial \varphi_{k}}{\partial X}+\left(\sum_{k=1}^{N}\left(\varphi_{k} \cdot v_{k}\right) \frac{\partial \varphi_{k}}{\partial Y}\right)\right] \varphi_{i} d x d y\right]$

$-\sum_{k=1}^{N} \theta_{k} \int_{A^{(e)}}\left[\frac{\partial \varphi_{i}}{\partial X} \frac{\partial \varphi_{k}}{\partial X}+\frac{\partial \varphi_{i}}{\partial Y} \frac{\partial \varphi_{k}}{\partial Y}\right] d x d y=0$

The finite element formulation of the dimensionless stream function is defined as

$\Omega=-\left(\frac{\partial^{2} \Psi}{\partial X^{2}}+\frac{\partial^{2} \Psi}{\partial Y^{2}}\right)$

Using the basis set interpolation function, the stream function over the domain of interest is discretized into finite elements having nodes $\mathrm{N}$ $\psi(x, y)=\sum_{k=1}^{N} \varphi_{k}(x, y) \Psi_{k}$

Applying the Galerkin finite element residual equations to (18) becomes

$R_{i}^{(3)}=\sum_{k=1}^{N} \Psi_{k} \int_{A^{(e)}}\left[\frac{\partial \varphi_{i}}{\partial X} \frac{\partial \varphi_{k}}{\partial X}+\frac{\partial \varphi_{i}}{\partial Y} \frac{\partial \varphi_{k}}{\partial Y}\right] d x d y-\int_{A^{(e)}} \sum_{k=1}^{N}\left(\varphi_{k} \cdot \Omega_{k}\right) \varphi_{i} d x d y=0$

The process is terminated with convergence criterion $\sqrt{\left[\sum\left(R_{i}^{(j)}\right)^{2}\right] \leq 10^{-6}}$

\subsection{Numerical Procedure}

The dimensionless nonlinear governing partial differential equations of the conservations of mass, momentum and energy equations are solved by applying the Galerkin weighted residual finite-element method. Using the developed code in COMSOL Multiphysics 5.0, the modified nonlinear equations are transformed into linear algebraic equations with the aid of Newton's iteration method while the fully coupled system is set to perform 50 iterations. With 784 mesh elements and 112 boundary elements, a total of 5772 degrees of freedom were solved using the stationary solver. It then repeatedly solved problems of buoyant flow in the cavities and analyzed different temperature profiles as well as the convective flow patterns. With the factor in error estimated as 400 , the convergence tolerance reaches $10^{-6}$. The iterative process is tuned for a fast, efficient solution using dimensionless parameters already defined and a Buosinessq approximation for the buoyant force. The results are presented for Rayleigh numbers (Ra) of $10^{4}, 10^{5}, 10^{6}, 10^{7}$ and $10^{8}$ in form of streamlines and isotherms plots for aspect ratios (AR) of $0.25,0.5,1.0$ and 2.0 respectively.

\subsection{Program Validation}

The program code was validated with some numerical studies in literatures in order to check on the accuracy of the numerical procedure employed in this study.

Table 1 Comparison of Galerkin Finite Element method with other

\begin{tabular}{|c|c|c|c|c|c|c|}
\hline$R a$ & $N u$ & $\begin{array}{l}\text { Presen } \\
t \text { work }\end{array}$ & $\begin{array}{l}\text { Mousa, } \\
(2010)\end{array}$ & $\begin{array}{l}\text { Barakos } \\
\text { and } \\
\text { Assimac } \\
\text { o, (1994) }\end{array}$ & $\begin{array}{l}\text { Davis, } \\
1983\end{array}$ & $\begin{array}{l}\text { Fusegi } \\
\text { et al, } \\
1991\end{array}$ \\
\hline \multirow{3}{*}{$10^{4}$} & $\begin{array}{l}\mathrm{Nu} \\
(\max )\end{array}$ & 3.524 & 3.531 & 3.539 & 3.528 & 3.652 \\
\hline & At y & 0.143 & 0.145 & 0.143 & 0.143 & 0.123 \\
\hline & $\begin{array}{l}\mathrm{Nu} \\
\text { (avg) }\end{array}$ & 2.242 & 2.245 & 2.245 & 2.243 & 2.230 \\
\hline \multirow{3}{*}{$10^{5}$} & $\begin{array}{l}\mathrm{Nu} \\
(\max )\end{array}$ & 7.742 & 7.716 & 7.636 & 7.717 & 7.795 \\
\hline & At y & 0.071 & 0.085 & 0.085 & 0.081 & 0.083 \\
\hline & $\begin{array}{l}\mathrm{Nu} \\
(\text { avg) }\end{array}$ & 4.516 & 4.512 & 4.510 & 4.519 & 4.646 \\
\hline \multirow{3}{*}{$10^{6}$} & $\begin{array}{l}\mathrm{Nu} \\
(\max )\end{array}$ & 18.981 & 17.500 & 17.442 & 17.925 & 17.670 \\
\hline & At y & 0.0357 & 0.0400 & 0.0368 & 0.0378 & 0.0379 \\
\hline & $\begin{array}{l}\mathrm{Nu} \\
\text { (avg) }\end{array}$ & 8.725 & 8.819 & 8.806 & 8.799 & 9.010 \\
\hline
\end{tabular}
numerical results. 
Table 1 shows the numerical results that compare the maximum local Nusselt number and average Nusselt number along the hot walls and the locations where they occur. The square cavity considered is heated at the left wall and cooled at the right wall, the top and bottom wall is kept insulated. As can be noticed from Table 1, the results from Galerkin finite element analysis provide an excellent agreement with numerical methods of Mousa, (2010); Barakos and Assimacopoulos, (1994); Davis, (1983); Fusegi et al,(1991) considered.

\section{RESULTS AND DISCUSIONS}

The results from the numerical simulation of natural convection in rectangular cavities with different aspect ratios considered are presented in Streamlines and Isotherm plots as shown in Figures 2-6. Circular pattern of single vortex were produced in correlation with the width of the cavity in Fig. 2. This single vortex can be attributed to the low transport of heated fluid due to low Rayleigh number. Consequently, the Isotherm plots indicate an almost parallel vertical lines to the differential heated walls due to flow induced by buoyancy. Therefore, at $\mathrm{Ra}$ of $10^{4}$, heat is being transferred principally by conduction through the fluid. The vertical plots further transforms to horizontal parallel lines as the AR increases suggesting that heat transfer by conduction decreases as the AR increases. In Fig. 3, the single vortex is observed in the cavity of $\mathrm{AR}=0.25$ and 0.5 which further breaks into 2 vortices in the cavity of $\mathrm{AR}=1$ and 2, the production of more vortices is a result of increase in relative magnitude flow in convection heat transfer. The Isotherm reveals that heat applied at right wall of the cavity gradually expanded to the cold region as the AR increases. Hence the fluid rises to the top insulated wall of the cavity due to increase in buoyancy force. Temperature changes along the hot wall are maximized at the top and reduce from top to bottom wall of the cavity. Therefore, heat transferred by convection from the right wall is a dominant feature at cavity of $\mathrm{AR}=2$.

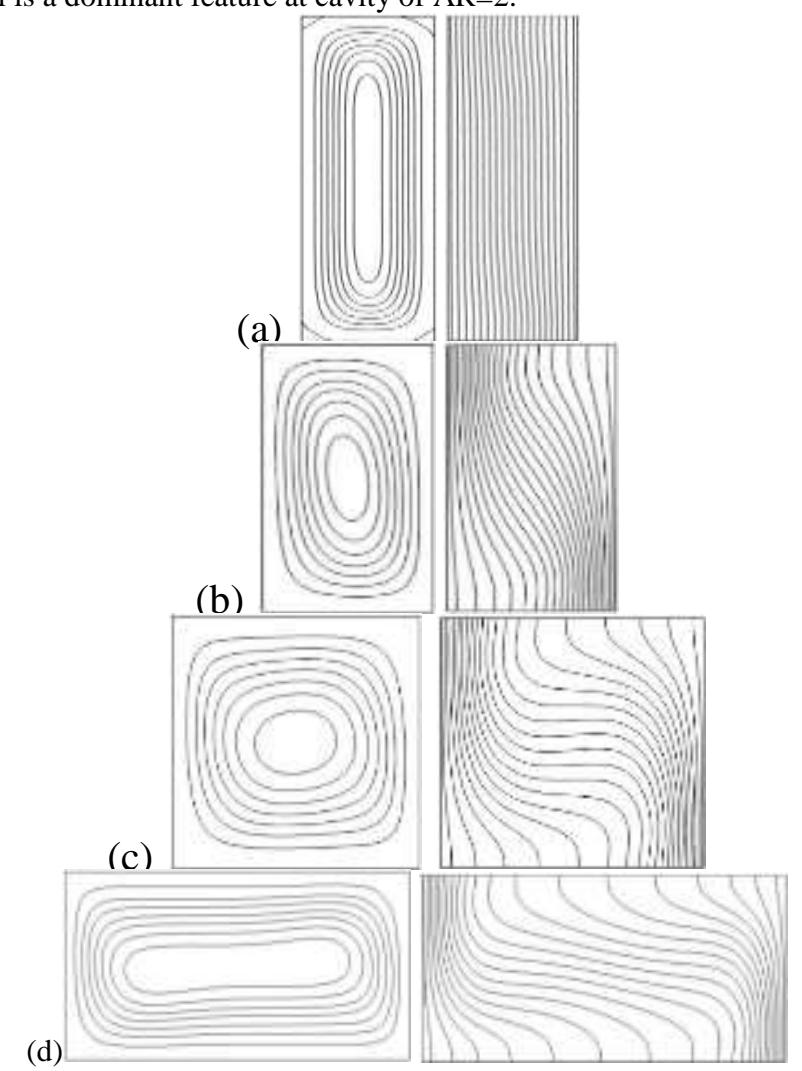

Fig. 2. Variation of Streamlines and Isotherms at $\mathrm{Ra}=10^{4}$ for (a) $\mathrm{AR}=0.25$, (b) $\mathrm{AR}=0.5$ (c) $\mathrm{AR}=1$ (d) $\mathrm{AR}=2$

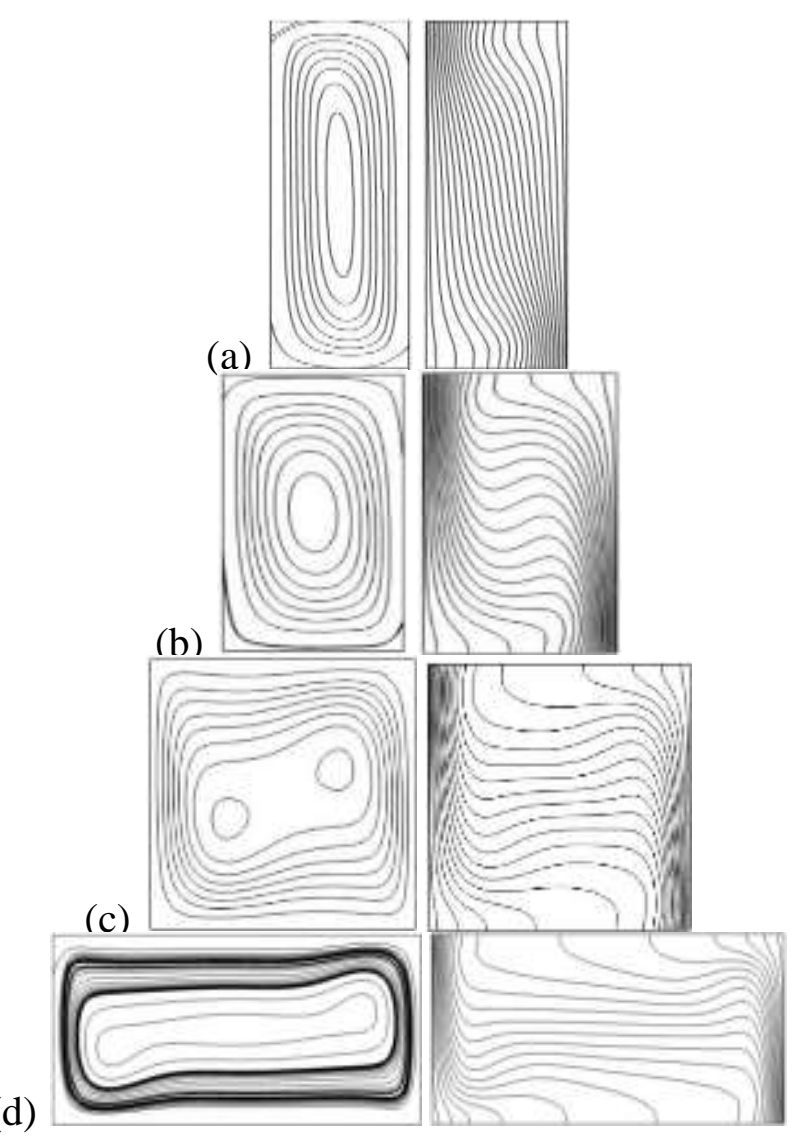

Fig. 3. Variation of Streamlines and Isotherms at $\mathrm{Ra}=10^{5}$ for (a) $\mathrm{AR}=0.25$, (b) $\mathrm{AR}=0.5$ (c) $\mathrm{AR}=1$ (d) $\mathrm{AR}=2$

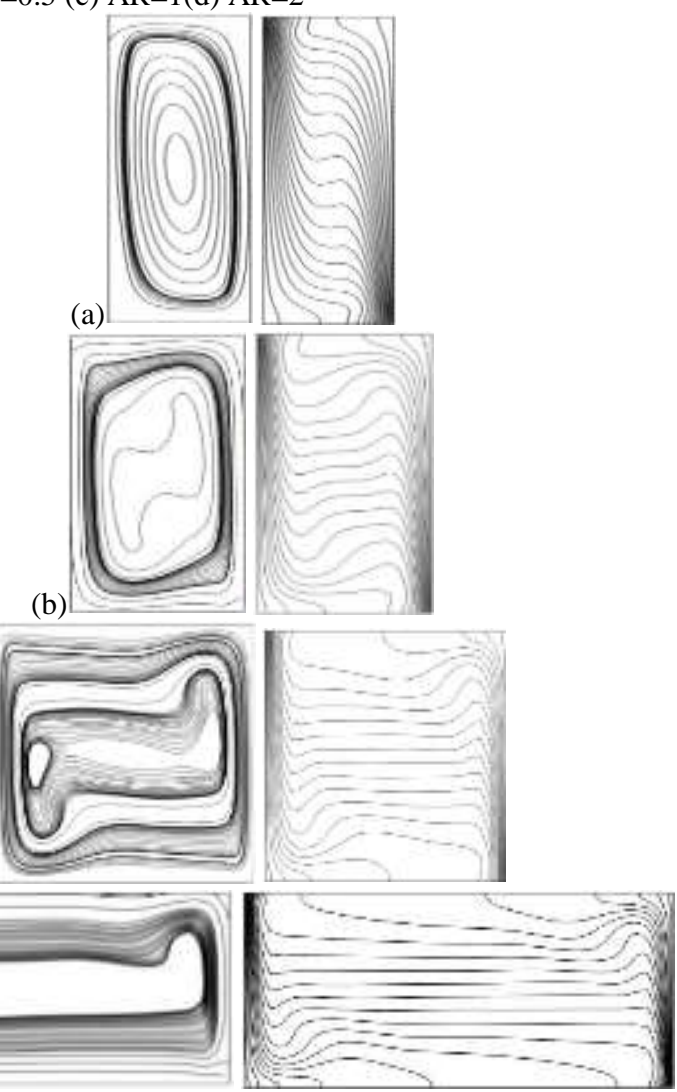

d)

Fig. 4. Variation of Streamlines and Isotherms at $\mathrm{Ra}=10^{6}$ for (a) $\mathrm{AR}=0.25$, (b) $\mathrm{AR}=0.5$ (c) $\mathrm{AR}=1$ (d) $\mathrm{AR}=2$ 
A closer view of Fig. 4 reveals more circular vortices are formed in the cavity for streamlines as the AR increases, the vortex lines thickens at the vertical side of the wall and spreads through the horizontal wall. These dominant thicken vortices can be attributed to increase in transport of hot fluid from the vertical right wall of the cavity. The Isotherm plots have increasingly transformed from vertical lines to horizontal lines from the hot wall to the cold wall when the AR is 2 , hence, the less dense fluid dominates the cavity as it increases in AR. Also observed in Fig. 5 is the flow fields represented by streamlines, temperature change between the vertical walls causes buoyant effects, closed streamlines are demonstrated by more recirculating vortices formed. Moreover, for streamlines, the vortices further breaks and increases with AR due to increase in Rayleigh number. Transport of the heated fluid is enhanced by the increased complex eddies formed at the center of the cavities while it gains more rupture at the cavity with $\mathrm{AR}$ of 2 . The Isotherms plots shows that an increased less dense fluid flowing from the hot right wall to the cold region becomes more dominant when the cavity AR is at maximum. As observed in Fig. 6, Circular vortices in cavity breaks and becomes more ruptured, heat transfer by convection has totally dominated the cavity when the $\mathrm{Ra}=10^{8}$ and $\mathrm{AR}=2$.

From observation of all the Figures 2-6, the system is in conduction mode when lower Rayleigh number $\mathrm{Ra}=10^{4}$ is applied and as the Rayleigh number increases, the effect of buoyancy forces is greatly increased, the system tends to become convection mode while the flow dynamics takes place, it changes the flowing pattern of the streamlines and Isotherms of the flow significantly which involves the shifting mode from conduction to convection for the heat transfer mechanism in the different aspect ratios considered, this is similar to Hassan et al, (2019) observations that at high Rayleigh numbers, there are changes in streamlines and isotherms contours.

(a)
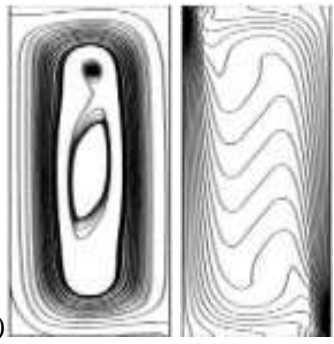

(b)
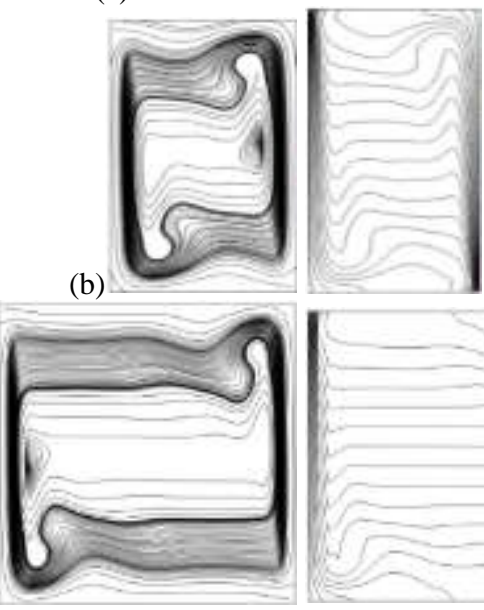

(c)

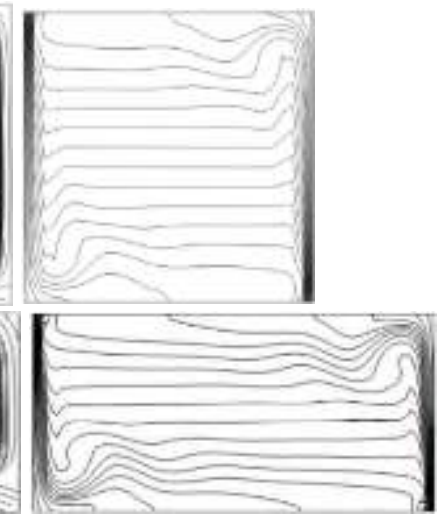

(d)

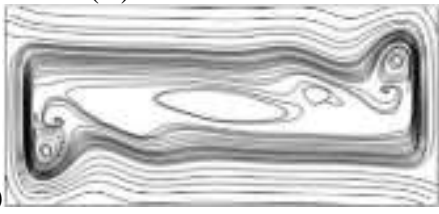

Fig. 5. Variation of Streamlines and Isotherms at $\mathrm{Ra}=10^{7}$ for (a) $\mathrm{AR}=0.25$, (b) $\mathrm{AR}=0.5$ (c) $\mathrm{AR}=1(\mathrm{~d}) \mathrm{AR}=2$ (c)

(b)

(a)
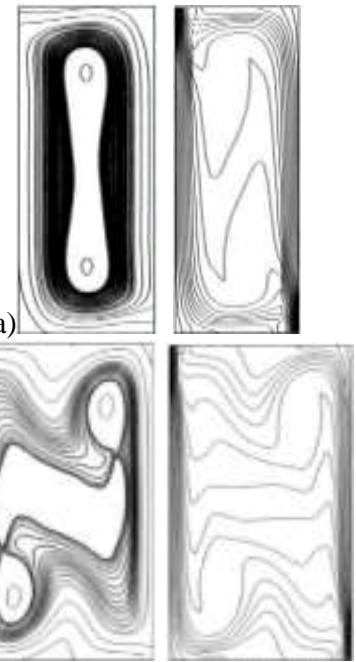

(d)
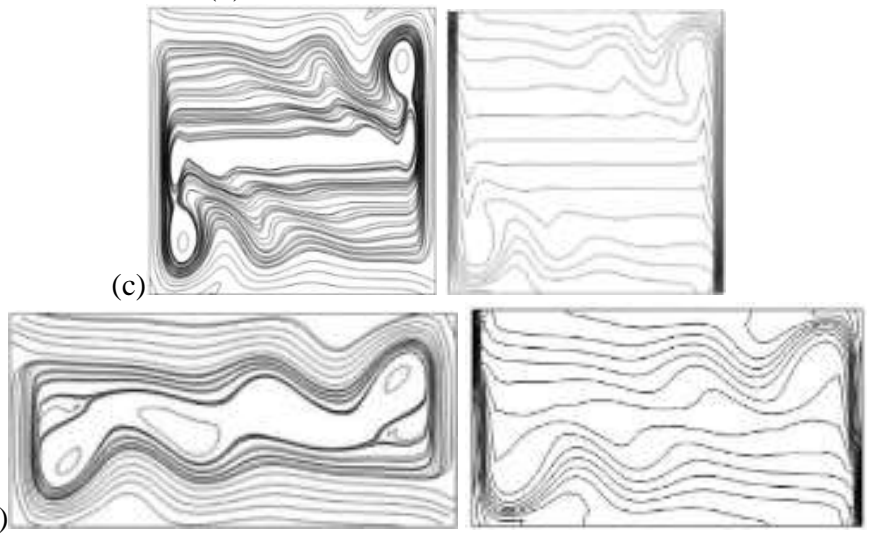

Fig. 6. Variation of Streamlines and Isotherms at $\mathrm{Ra}=10^{8}$ for (a) $\mathrm{AR}=0.25$, (b) $\mathrm{AR}=0.5$ (c) $\mathrm{AR}=1$ (d) $\mathrm{AR}=2$

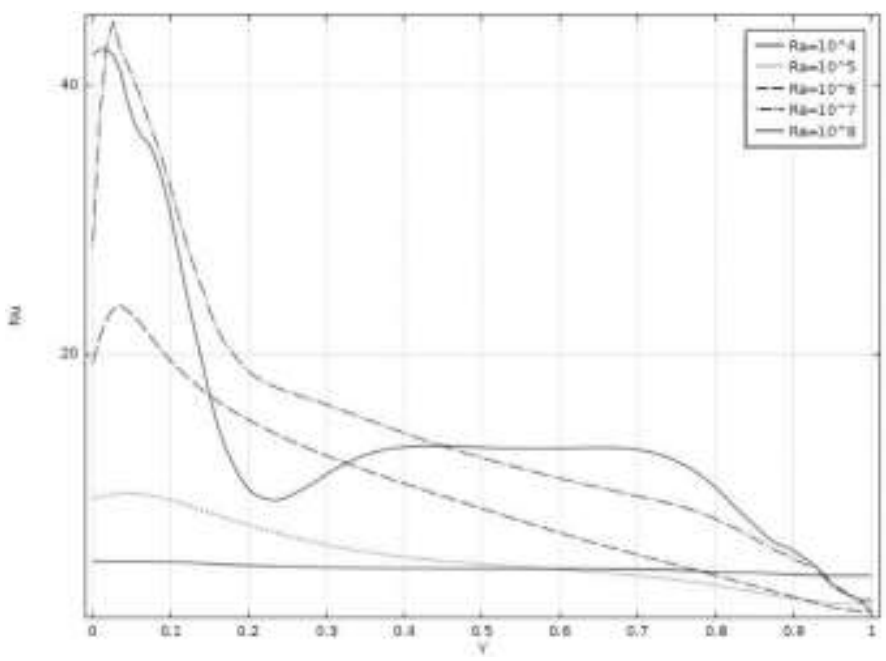

Fig. 7. Variation of Nusselt along the hot wall with varying Ra for $\mathrm{AR}=0.25$

Figures 7 and 8 show the variation of vertical hot wall distance as a function of Nusselt number for various $\mathrm{Ra}$. It worth noting that maximum local Nusselt number is observed at a location closed to the lower side of the hot vertical wall as the Rayleigh number increases. 


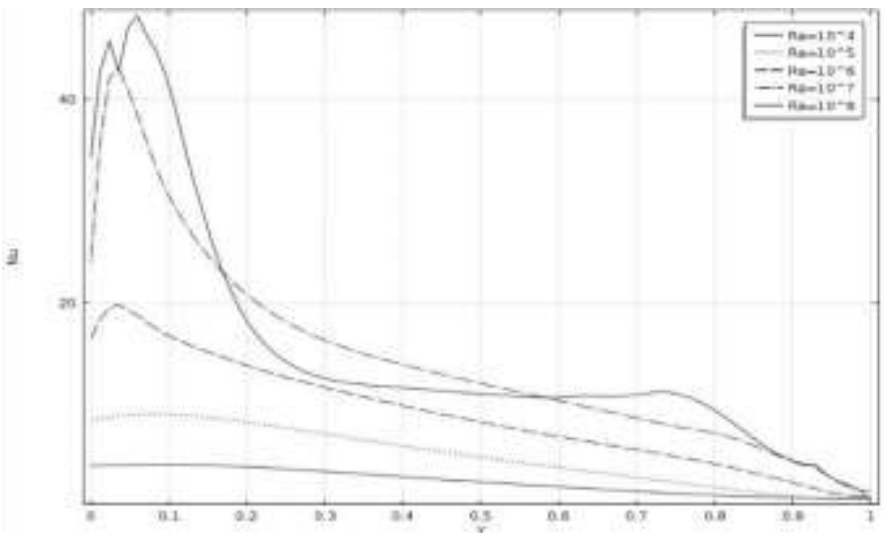

Fig. 8. Variation of Nusselt along the hot wall with varying Ra for $\mathrm{AR}=0.5$

The result in Fig. 7 indicates that heat is being transferred by convection at the boundary of the hot wall as Rayleigh number increases. However, temperature gradients towards the left cold side of the cavity decreases indicating a reduced heat transfer by conduction attributed to cold region of the cavity. With an increase in AR as observed in Fig. 8, maximum local Nusselt number variation extends along the left wall of the cavity thereby leading to conduction dominant heat transfer rate. Moreover, Fig. 9 shows the correlation between Nusselt number and Rayleigh number for AR 0.25, 0.5, 1 and 2. The results demonstrated that the total heat transfer depends on the Nusselt number which is due to the influence of Rayleigh number inside the cavity, hence, increase in the Rayleigh number promotes the flow and convective heat transfer Elyazid et al, (2021).It is also observed that cavities with smaller aspect ratios exhibit lower rate of heat transfer by conduction. The data obtained established that AR generally increases with increase in Nusselt number as Rayleigh number increases.

Figure 10 presents the variation of Nusselt number with the aspect ratios. The results reveal that at a fixed aspect ratio, Nusselt number increases with increase in Rayleigh number.

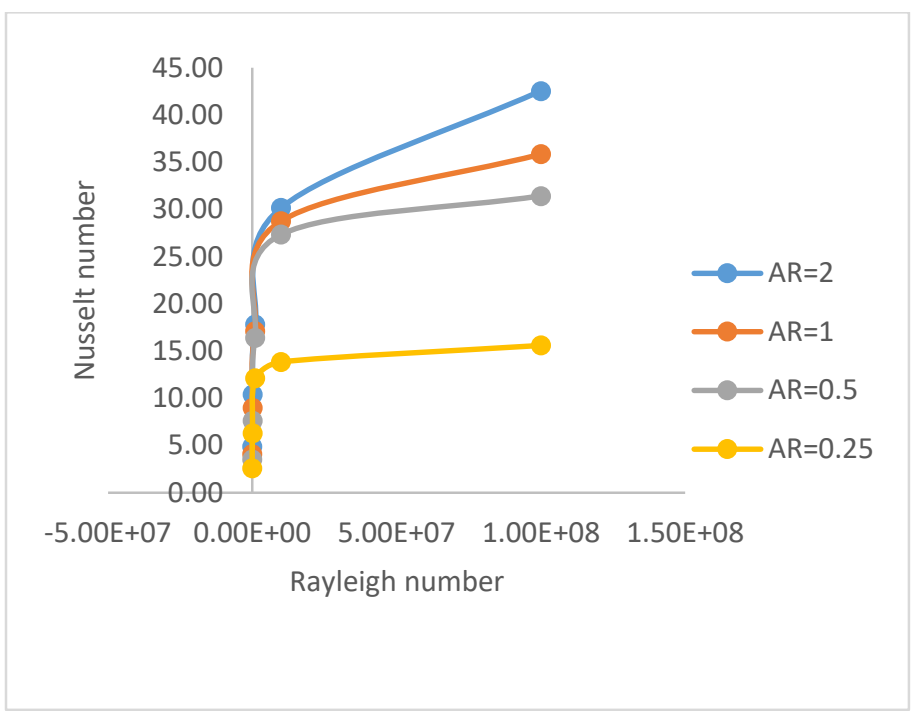

Fig. 9. Correlation between Nusselt number and Rayleigh numbers for various Aspect ratios.

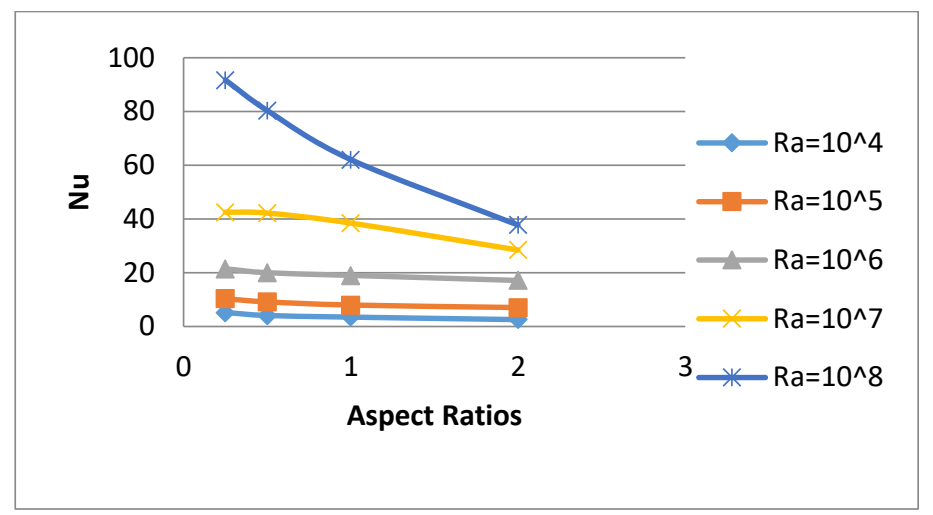

Fig. 10. Variation of Nusselt number with the Aspect ratios

Some correlations with the Nusselt numbers to aspect ratios $1<\mathrm{X} / \mathrm{Y}<10$ have been cited by Incropera et al, (2007) as follows;

$$
\begin{gathered}
N u=0.22\left[\frac{P r}{0.2+P r} R a\right]^{0.28}\left(\frac{X}{Y}\right)^{-\frac{1}{4}} \\
{\left[\begin{array}{c}
2<\frac{X}{Y}<10 \\
P r<10^{5} \\
10^{3}<\operatorname{Ra}<10^{10}
\end{array}\right]}
\end{gathered}
$$

The empirical correlation is written in the form

$$
N u=C R a^{n}
$$

Using the value of $\mathrm{Pr}=0.71$ for Aspect ratio $(\mathrm{X} / \mathrm{Y})=0.25,0.5,1$ and 2 . The following results in Table 2 were obtained and then compared with result of the simulation in this study. It could be observed that, the simulated equation approximately agrees with those empirical correlation equations suggested in Incropera et al, (2007), the simulated equations obtained presents a good fit with a correlation coefficient approximately 0.91 .

Table 2 Comparison of Empirical correlations with simulated correlation equations

\begin{tabular}{|c|c|l|c|}
\hline AR/Studies & $\begin{array}{l}\text { Incropera, et } \\
\text { al., 2007 }(\mathrm{Nu})\end{array}$ & $\begin{array}{l}\text { Present work } \\
(\mathrm{Nu})\end{array}$ & $\begin{array}{l}\text { Correlation } \\
\text { Coefficient } \\
\left(R^{2}\right)\end{array}$ \\
\hline $\mathrm{AR}=0.25$ & $0.290 \mathrm{Ra}^{0.28}$ & $0.210 \mathrm{Ra}^{0.2761}$ & 0.9280 \\
\hline $\mathrm{AR}=0.5$ & $0.244 \mathrm{Ra}^{0.28}$ & $0.196 \mathrm{Ra}^{0.2771}$ & 0.9091 \\
\hline $\mathrm{AR}=1$ & $0.244 \mathrm{Ra}^{0.28}$ & $0.148 \mathrm{Ra}^{0.2951}$ & 0.9285 \\
\hline $\mathrm{AR}=2$ & $0.173 \mathrm{Ra}^{0.28}$ & $0.875 \mathrm{Ra}^{0.3221}$ & 0.8925 \\
\hline
\end{tabular}

\section{CONCLUSIONS}

The numerical simulation of natural convection in rectangular cavities with different aspect ratios has been performed using Galerkin based finite element method in order to determine the effects of Rayleigh numbers (Ra) at $10^{4}, 10^{5}, 10^{6}, 10^{7}, 10^{8}$ and aspect ratios (AR) of 0.25 , $0.5,1$ and 2 on flow profile and heat transfer coefficient. The emanated results are summarized as follows;

(i) Heat transfer mechanisms, flow behaviour and temperature distributions for various aspect ratios were strongly influenced while varying Rayleigh numbers from $10^{4}$ to $10^{8}$. At a fixed aspect ratio (AR), increased in Rayleigh number causes the streamlines to 
become thicker close to the walls of the cavity, indicated an enhanced rate of heat transfer by convection.

(ii) At a constant Rayleigh number, the thickness of the thermal boundary layers in the cavities was dramatically increased as the aspect ratio increases.

(iii) Buoyancy driven force is increased when the Rayleigh number increases within the cavity. As Rayleigh number increases, flow fields became closely packed within the cavity, the temperature gradient between vertical walls are as a result of buoyancy effects. For aspect ratio of 2 , the Rayleigh number at $10^{8}$ reported the highest buoyancy driven force.

(iv) Furthermore, empirical correlations obtained within the cavities of AR varied from $0.25,0.5,1$ and 2 for Nusselt number presented a good fit of approximately 0.91 coefficient of regression. This demonstrated the behavior of Nusselt number in relation to Rayleigh number and Aspect ratios in rectangular cavities.

\section{ACKNOWLEDGEMENTS}

Nil.

\section{NOMENCLATURE}

$\begin{array}{ll}u & \text { Velocity in x-direction }(\mathrm{m} / \mathrm{s}) \\ v & \text { Velocity in y-direction }(\mathrm{m} / \mathrm{s}) \\ R a & \text { Rayleigh number } \\ P r & \text { Prandtl number } \\ \propto & \text { Thermal diffusivity }(\mathrm{W} / \mathrm{m} \cdot \mathrm{K}) \\ g & \text { Acceleration due to gravity }\left(\mathrm{m} / \mathrm{s}^{2}\right) \\ T_{\mathrm{c}} & \text { Cold temperature }(\mathrm{K}) \\ T_{\mathrm{h}} & \text { Hot temperature }(\mathrm{K}) \\ \beta & \text { Volumetric thermal expansion }\left(\mathrm{K}^{-1}\right) \\ \delta & \text { Width of the cavity }(\mathrm{m}) \\ \mathrm{L} & \text { Height of the cavity }(\mathrm{m}) \\ P & \text { Pressure }(\mathrm{Pa}) \\ \mathrm{Nu} & \text { Nusselt number }\end{array}$

Greek Symbols

$P \quad$ Density $\left(\mathrm{kg} / \mathrm{m}^{3}\right)$

$\varphi \quad$ Interpolation function

$\mathrm{R}^{\mathrm{n}} \quad$ Residual function

$\Psi \quad$ Stream function

$\Omega \quad$ Vorticity

$\vartheta \quad$ Dynamic viscosity $(\mathrm{Kg} . \mathrm{s} / \mathrm{m})$

$\begin{array}{cr}\text { Subscripts } \\ c & \text { cold } \\ h & \text { hot }\end{array}$

\section{REFERENCES}

Abdulkadhim A, Abed A.M, Al-Farhany K., 2018,"Computational investigation of conjugate heat transfer in cavity filled with saturated porous media", Frontiers in Heat and Mass Transfer, 11. https://doi.org/10.5098/HMT.11.12

Ali ME-S, Nuhait A. O, Alabdulkarem A, Almuzaiqer R.,2018, ” Free convection heat transfer inside square water-filled shallow enclosures" PLoS ONE 13(10): e0204251. https://doi.org/10.1371/journal.pone.0204251
Aydin, O. and Yang, J., 2000, "Natural Convection in Enclosures with Localized Heating from Below and Symmetrically Cooling from Sides" Int. J. Numerical Methods Heat Fluid Flow, Vol. 10, pp. 518-529.

https://doi.org/10.1108/09615530010338196

Barakos G, Mitsoulis E and Assimacopoulos D., 1994, "Natural Convection flow in a square cavity revisited: Laminar and turbulent models with wall functions" Int. J. Num. Meth. Fluids 18, 695-719. https://doi.org/10.1002/fld.1650180705

Barik AL-Muhja and Khaled Al-Farhany., 2019, "Numerical Investigation of the effect of baffle inclination angle on nanofluid natural convection heat transfer in a square enclosure" Al-Qadisiyah Journal for engineering sciences 12, 061-071. https://doi.org/10.30772/qjes.v12i2589

Davis, G. De Vahl., 1983, "Natural Convection of air in a square cavity: A benchmark numerical solution" Int. J. Num. Meth. Fluids 3, 249-264.

https://doi.org/10.1002/fld.1650030305

D. Santhi Kumari, Venkat Subrahmanyam Sajja, P.M. Kishore., 2021. "Numerical solution of the effects of heat and mass transfer on unsteady MHD free convection flow past an infinite vertical plate". Frontiers in Heat and Mass Transfer (FHMT), 16,24. http://dx.doi.org/10.5098/hmt.16.24

Elyazid Flilihi, Mohammed Sriti, Driss Achemlal, Mohamed El harmi, 2021, "CFD analysis of free convection in non-Darcian porous medium and comparison with similarity approach". Frontiers in Heat and Mass Transfer (FHMT), 17,7. http://dx.doi.org/10.5098/hmt.17.7

Fusegi T, Hyun J. M, Kuwahara K and Farouk B, 1991, “A numerical study of three-dimensional natural convection in a differentially heated cubical enclosure" Int. J. Heat Mass Transfer 34, 1991, 1543-1557. https://doi.org/10.1016/0017-9310(91)90295-P

Hassa Sh.Majdi, Amma Adulkadhim, Azher M.Abed., 2019. "Numerical Investigation of Natural convection heat transfer in a parallelogramic enclosure having an inner circular cylinder using liquid nanofluid". Frontiers in Heat and Mass Transfer (FHMT), 12,2 .

http://dx.doi.org/10.5098/hmt.12.2.

Incropera, DeWitt, Bergman, Lavine, 2007," Fundamentals of Heat and Mass Transfer". Sixth Edition.John Wiley \& Sons, Inc.

Lo D C., Young, D. L, Tsai C.C., 2004, "High resolution of 2D natural convection in cavity by DQ method" Journal of Computational and Applied Mathematics; 203:219-236. https://doi.org/10.1016/j.cam.2006.03.021

Mahmud H. Ali, Rawand E. Jalal, 2020, "Natural convection in a Square enclosure with different openings and involves two cylinders: A numerical approach", Frontiers in Heat and Mass Transfer (FHMT), 15, 27. http://dx.doi.org/10.5098/hmt.15.27.

Mazgar, A., Hajji, F., Jarray, K., Ben Nejma, F, 2019, “Conjugate non-Gray Gas Radiation Combined with Natural Convection inside a Square Cavity with Internal Heat Source: Entropy Generation" Conference, 6th International Conference on Green Energy and Environmental Engineering, April 27-29th, Tabarka, Tunisia. 
Mohamed Loukili, Kamila Kotrasova, Denys Dutykh.,2020, “A Computational simulation of steady natural convection in an $\mathrm{H}$-form cavity", 4th computational methods in systems and software 2020, Szczecin, Poland.

https://hal.archives-ouvertes.fr/hal-02615655

Mousa, M.M., 2010, "Modeling of Laminar Buoyancy Convection in a square Cavity Containing an Obstacle", Mathematics Subject Classification: 65M60, 76D05, 80A20. Benha University. https://doi.org/10.1007/S40840-015-0188-Z

Patil A. B. and Singh S. N., 2017, "Numerical Study of Natural Convection in Tiny Closed Cavities with Different Aspect Ratios", Imperial Journal of Interdisciplinary Research (IJIR) Vol-3, Issue4, ISSN: 2454-1362.

http://www.onlinejournal.in

Raisi, A., Arvin, A., 2018, “A numerical study of the effect of fluidstructure interaction on transient natural convection in an air-filled square cavity", International Journal of Thermal Sciences, 128, pp.
$1-14$.

http://dx.doi.org/10.1016/j.ijthermalsci.2018.02.012

Saddam Atteyia Mohammad, 2020. "Effects of variable viscosity on heat and mass transfer by MHD mixed convection flow along a vertical cylinder embedded in a non-Darcy porous medium". Frontiers in Heat and Mass Transfer (FHMT), 14, 7. http://dx.doi.org/10.5098/hmt.14.7.

Selimefendigil F, Öztop H.F., 2016, "Conjugate natural convection in a cavity with a conductive partition and filled with different nanofluids on different sides of the partition" Journal of Molecular Liquids, 216, 67-77.

https://doi.org/10.1016/j.molliq.2015.12.102

Souda Morsli, Amina Sabeur, Mohammed El Ganaoui., 2017, "Influence of aspect ratio on the natural convection and entropy generation in rectangular cavities with wavy-wall", Energy Procedia. Vol 139, December, pp 29-36. https://doi.org/10.1016/j.egypro.2017.11.168 